\title{
Role of Cardiac Magnetic Resonance (CMR) Imaging in the Assessment of Wall Motion Abnormalities in Myocardial Infarction Patients
}

\author{
MAGDY M. ELRAKHAWY, M.D.*; MONA K.E. SALAMA NASSAR, M.Sc.*; NEHAD M.S. FOUDA, M.D.*; \\ SHADY H. MOHAMED, M.D.** and AHMED M. ABD EL KHALEK, M.D.*
}

The Department of Diagnostic \& Interventional Radiology, Faculty of Medicine, Mansoura University* and Cardiology Unit, Mansoura University Specialized Internal Medical Hospital**, Mansoura, Egypt

\begin{abstract}
Background: Subtle changes in left ventricular wall motion patterns have diagnostic and prognostic values in patients with Coronary Artery Disease (CAD).

Aim of Study: The aim of this studywasto highlightthe diagnostic role of Cardiac Magnetic Resonance imaging ( $\mathrm{CMR}$ ) in assessment of the myocardial wall motion abnormalities in Myocardial Infarction (MI) patients.
\end{abstract}

Material and Methods: This retrospective research was performed in 50 patients classified into two groups: Group 1 (25 patients with Acute Myocardial Infarction (AMI) (23 males and 2 females) and group 2 (25 patients with Chronic Myocardial Infarction (CMI) (22 males and 3 females). Both classes were diagnosed based on medical, echocardiography, and other approaches of cardiologist investigation. The mean age was $52.16 \pm 6.884$ years for group 1 and $51.04 \pm 12.341$ years in group 2. All patients underwent CMR evaluation using Cine Steady State Free Precession (SSFP) in short axis and 4 chamber views.

Results: Hypokinesia was the most commonly observed wall motion abnormality among the study groups. It was seen in $13(52 \%)$ patients in group 1 and the same in group 2 . Akinesia was seen in $12(48 \%)$ patients in group 1 and 10 (48\%) patients in group 2 and dyskinesia was observed in 2 ( $8 \%$ ) patients in group 2 and not observed in group 1.

Conclusion: Identification of wall motion abnormalities in MI patients may help to identify higher risk patients that would benefit from an earlier invasive strategy. We conclude that CMR detected the wall motion abnormality precisely and should be added to the routine investigation of MI patients for better evaluation and assessment.

Key Words: CMR - WMA-Heart.

Correspondence to: Dr. Mona Kamal

Nassar,E-Mail: mona_nassar85@ mans.edu.

\section{Introduction}

ONE of the most common causes of death in the world is Coronary Artery Disease (CAD) [1]. For certain heart conditions, echocardiography is the first-line cardiac imaging trial [2]. It is readily available and provides material that is structural and functional. However, echocardiography has some drawbacks [2]. Cardiac Magnetic Resonance imaging (CMR) has been established as an imaging method that provide echocardiography with additional and supportive information [2]. It offers cross-sectional images of great quality that promote accurate anatomical description [2]. It also makes extremely reproducible estimates of the changes of ventricular volume and wall motion [2]. MR images are generated using a Steady State Free Precession (SSFP) sequence with a good discrimination between the blood system and the myocar-

\footnotetext{
Abbreviations:

AHA : American Heart Association. AMI : Acute Myocardial Infarction. CAD : Coronary Artery Disease. CMI : Chronic Myocardial Infarction. CMR: Cardiac Magnetic Resonance. ECG: Electrocardiography.

FA : Fractional Anisotropy. FOV : Field of View.

IRB : Institutional Research Board.

MI : Myocardial Infarction.

MR: Magnetic Resonance.

MRI: Magnetic Resonance Imaging.

SA : Short Axis

SPSS : Statistical Package of Social Sciences.

SSFP: Steady State Free Precession.

$T$ : Tesla.

TE : Echo Time

TR : Repetition Time.
} 
dium [3]. With superior endocardial boundary determination, Cine (MRI) promotes functional imaging of cardiac wall motion, which enables more accurate measurement of wall motion [3].

\section{Material and Methods}

This retrospective study was performed at the Department of Diagnostic Radiology in Mansoura University Hospitals, between September 2017 and February 2020. The study population included in this study were 50 patients who were referred by cardiologists of Cardiology Department in Mansura University Internal Medicine Hospital for cardiac MRI examination. Written consent was taken from all patients and this study accepted by Institutional Research Board (IRb) committee. This study enrolled, 45 males (90\%) and 5 females ( $10 \%$ ) with an age range between 25-70 years with mean age $51.60 \pm 9.906$. The study group was classified as group 1 including 25 AMI (23 males, 2 females) patients and group 2 including 25 CMI ( 22 males, 3 females) patients. The mean age was 52 . $16 \pm 6.884$ years for group 1 and 51.04 \pm 12.341 years in group 2. Each patient has been assigned to history taking, laboratory examinations and CMR. Patients with chronic ventricular arrhythmias, uncooperative patients, and patients with MRI contraindications were excluded from our research. Written permits were granted to all patients.

\section{CMR:}

A $1.5 \mathrm{~T}$ (Ingenia, Philips, the Netherlands) magnetic resonance scanner has been used to perform CMR imaging. A 16-channel torso phasedarray receiver coil was used to transmit signals. All data acquisition was performed retrospectively by ECG.

\section{Protocol for CMR acquisition:}

In the following sequence, the scanning procedure was performed: Scout images (axial, coronal and sagittal) (acquisition matrix $220 \mathrm{X} \mathrm{176}$, slice length $10 \mathrm{~mm}$, flip angle $50^{\circ}$, voxel size $1.6 \mathrm{~mm} \times 1$. $9 \mathrm{~mm} \times 10 \mathrm{~mm}$, FOV $450 \times 450 \mathrm{~mm}^{2}$ and echo/ repetition time (TE/TR) shortest. Sequences accumulated on the Cine Steady State Free Precession ( SSFP): Four chambers and short axis (acquisition matrix $220 \times 176$, FOV $350 \times 350 \mathrm{~mm}^{2}$, flip angle $60^{\circ}$ voxel size $1.6 \mathrm{~mm} \times 1.9 \mathrm{~mm} \times 10 \mathrm{~m}$, echo/ repetition time (TR/TE) 6.1/3 msand slice thickness 6-8mm). Then there is a "stack" of contiguous SSFP cine images with the same technical criteria obtained along the short axis, including the whole ventricle from base to apex.
Analysis and interpretation of cine SSFP images:

The anatomy and function of the left ventricle is quantitatively tested on the images of the short axis view. The myocardium was segmented into 17 segments according to American Heart Association (AHA) model and each segment was assess in the end systolic and end diastolic images for evaluation of the wall motion abnormalities.

\section{Analysis of statistics:}

The data collection and statistical analyses were carried out using SPSS version 21 (Statistical Package of Social Sciences). The results were expressed as number and percentage. The data normality was first tested using a single-sample test.

\section{Results}

This research involved 50patients, classified as: Patients with AMI (group 1, 25 patients, 23 males and 2 females) and patients with CMI (group 2, 25 patients 22 males and 3 females). The mean age of group 1 was $52.16 \pm 6.8$ years and the mean age of group 2 was $51.04 \pm 12.3$ years.

Table (1) shows that hypokinesia was the most commonly observed wall motion abnormality among the study groups. It was seen in 13 (52\%) patients in group 1 and the same in group 2. Akinesia was seen in $12(48 \%)$ patients in group 1 and $10(48 \%)$ patients in group 2 and dyskinesia was observed in $2(8 \%)$ patients in group 2 and not observed in group 1. There was no statistically significant difference among the study groups as regard wall motion abnormalities with $p$-value 0 . 33.

Table (2) shows that the most affected segment by wall motion abnormalities was the mid anteroseptal $18(12 \%)$ followed by the apical septal 17 ( $11.4 \%$ ), then the mid inferior and inferosptal segments $14(9.4 \%)$. The number of segments is more than the number of patients due to overlapping of some segments in cases.

Table (1): Wall motion abnormalities among the study groups.

\begin{tabular}{llll}
\hline & $\begin{array}{l}\text { Group 1 } \\
(\mathrm{No}=25)\end{array}$ & $\begin{array}{l}\text { Group 2 } \\
(\mathrm{No}=25)\end{array}$ & $\begin{array}{c}p- \\
\text { value }\end{array}$ \\
\hline Akinesia & $12(48 \%)$ & $10(40 \%)$ & 0.33 \\
Hypokinesia & $13(52 \%)$ & $13(52 \%)$ & 0.33 \\
Dyskinesia & $0(0 \%)$ & $2(8 \%)$ & 0.33 \\
\hline
\end{tabular}

Data expressed as frequency (No-\%).

$*$ : Significance $<0.05$. 

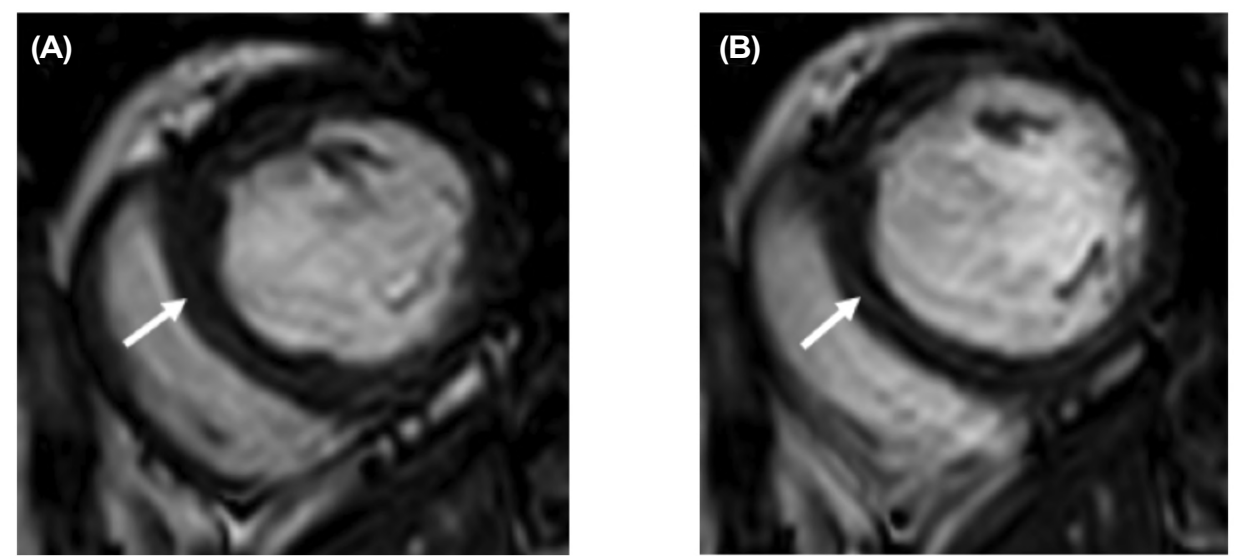

Fig. (1): A known case of chronic myocardial infarction along LAD and LCX territories. (A,B) Cine Steady-State Free Precession (SSFP) short axis end systolic (A) and end diastolic (B) Images show a kinetic mid anteroseptal myocardial segment (white arrows).
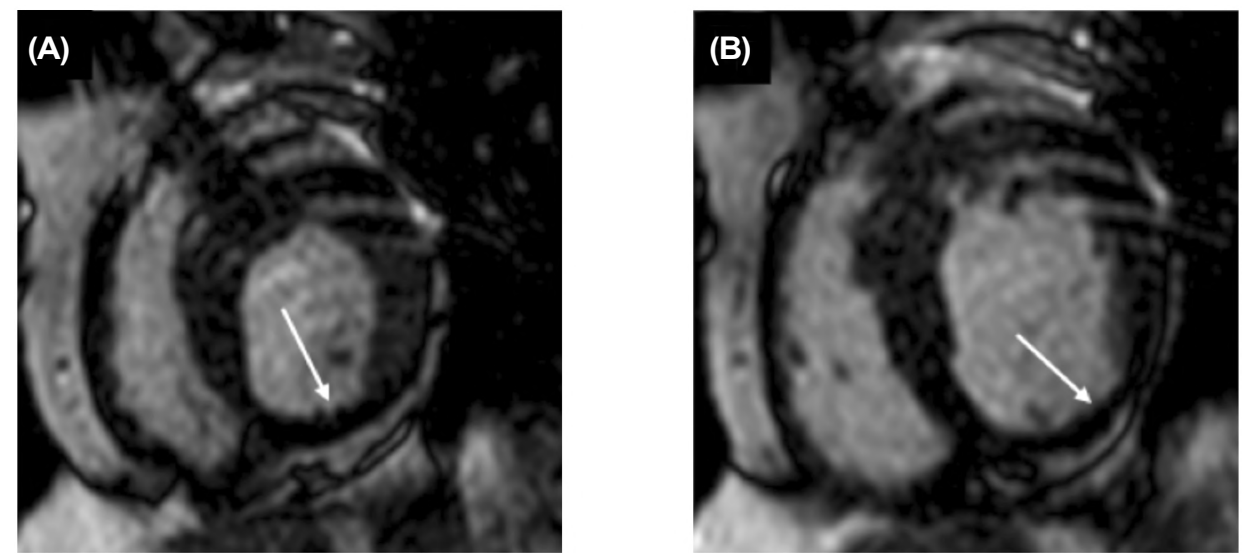

Fig. (2): A known case of chronic myocardial infarction along RCA territory. (A,B) Cine Steady-State Free Precession (SSFP) short axis end systolic (A) and end diastolic (B) Viewsshow dilated left ventricle with a kinetic mid inferior myocardial segment associated with myocardial thinning (thickness $<5 \mathrm{~mm})$ ( white arrows).

Table (2): The involved myocardial segments by wall motion abnormalities among the study groups.

\begin{tabular}{lll}
\hline & No & Percent \\
\hline 1- Basal anterior & 3 & $2 \%$ \\
2- Basal anteroseptal & 5 & $3.3 \%$ \\
3- Basal inferoseptal & 8 & $5.4 \%$ \\
4- Basal inferior & 12 & $8.1 \%$ \\
5- Basal inferolateral & 4 & $2.7 \%$ \\
6- Basal anterolateral & 0 & $0 \%$ \\
7- Mid anterior & 11 & $7.4 \%$ \\
8- Mid anteroseptal & 18 & $12 \%$ \\
9- Mid inferoseptal & 14 & $9.4 \%$ \\
10- Mid inferior & 14 & $9.4 \%$ \\
11- Mid inferolateral & 9 & $6 \%$ \\
12- Mid anterolateral & 3 & $2 \%$ \\
13- Apical anterior & 13 & $8.7 \%$ \\
14- Apical septal & 17 & $11.4 \%$ \\
15- Apical inferior & 8 & $5.4 \%$ \\
16- Apical lateral & 2 & $1.3 \%$ \\
17- Apex & 7 & $4.7 \%$ \\
\hline
\end{tabular}

Data expressed as frequency (No-\%).

\section{Discussion}

CAD is the primary source of morbidity and mortality worldwide. The most popular type of CAD is MI [4]. A secondary death of myocardial cells due to sustained ischemia is known as MI [5]. In patients with CAD, slight variations in left ventricular wall motion patterns have diagnostic and prognostic values [6]. The identification of wall motion abnormalities in these patients will help to identify patients at higher risk who may benefit from an earlier invasive approach. WMA will occur within seconds of myocardial ischemia onset [7]. In this study, WMA were assessed in all patients using SSFP cine short axis and 4 chamber images. The most common type of wall motion abnormality among our study groups was the hypokinesia. It was observed in $13(52 \%)$ patients in group 1 and the same in group 2. The second most common abnormality was akinesia which was detected in $12(48 \%)$ patients in group 1 and 10 ( $40 \%$ ) patients in group 2 followed by dyskinesia 
which was observed in 2 patients $(8 \%)$ in group 2 and not seen in group 1 . These findings were parallel to the study of Al-Gharbawi and Saeed, [ 8], who studied 101 patients with MI. Patients were grouped according to segments of regional wall motion abnormalities into 3 groups: Group A ( segments $1,2,7,8,13,14$ ), group B (segments $3,4,9,10,15$ ), and group $C$ (segments $5,6,11$, $12,13,16)$. They found that $(47.5 \%)$ of patients in group A had hypokinesia, Akinesia in (14.9\%), and dykinesia in $(21.8 \%)$. (44.4\%) of patients in group (B) had regional wall motion abnormality in form of hypokinesia (35.6\%), akinesia (7.9\%), and dyskinesia(1\%). (27.8\%) of patients in group (C) showed hypokinesia, (5\%) Akinesia, and (8\%) dyskinesia, majority were associated with RCA lesions in form of critical and total occlusions. In contrast to our results, Shivpuje Anjali and Page [ 9] reported that transmural MI is associated with akinesia or dyskinesiaand that hypokinesis is nonspecific and is less significant in distinguishing between injured and uninjured myocardium. These may be due to the use of 2D echocardiography in the assessment which us less accurate than CMR.

In the analysis of WMA, it is important to determine which myocardial segment was affected according to AHA 17 model. Our results showed that, the most commonly affected segment by wall motion abnormalities was the mid anteroseptal ( $12 \%)$ followed and the apical septal $(11.4 \%)$, then the mid inferior and inferosptal segments 14 (9.4\%), these findings were in concordance with Codreanu et al., [6], who found that the most affected myocardial segment was the mid anteroseptal segment ( $12 \%$ ) followed by the apical septal segment ( $11.4 \%)$.

\section{Conclusion:}

In summary, we share points of similarity with the previously compared studies that CMR can be used for better assessment and precise evaluation of the myocardial wall motion abnormalities in post myocardial infarction patients. It also helps in identifying higher risk patients that would benefit from an earlier invasive strategy.

\section{Limitations:}

Small sample size. No availability of myocardial tagging or T1 mapping images.

\section{References}

1- SAEED M., VAN T.A., KRUG R., HETTS S.W., WILSON M. W. J.C.D. and THERAPY: Cardiac MR imaging: Current status and future direction. Cardiovascular Diagnosis and Therapy, 5 (4): 290-310, 2015.

2- STOKES M.B., NERLEKAR N., MOIR S. and TEO K.S. J.A.F.P.: The evolving role of cardiac magnetic resonance imaging in the assessment of cardiovascular disease. Australian Family Physician, 45 (10): 761, 2016.

3- BARITUSSIO A., SCATTEIA A. and BUCCIARELLIDUCCI C.: Role of cardiovascular magnetic resonance in acute and chronic ischemic heart disease. The international journal of cardiovascular imaging, 34 (1): 67-80, 2018.

4- JAYARAJ J.C., DAVATYAN K., SUBRAMANIAN S. and PRIYA J.: Epidemiology of Myocardial Infarction. In Davatyan, K. (ed.), Myocardial Infarction (pp. 9-20). Intech Open, London, UK, 2018.

5- RAJIAH P., DESAI M.Y., KWON D. and FLAMM S.D.: MR imaging of myocardial infarction. Radiographics, 33 (5): 1383-412, 2013

6- CODREANU I., PEGG T.J., SELVANAYAGAM J.B., ROBSON M.D. and RIDER O.J.: Comprehensive Assessment of Left Ventricular Wall Motion Abnormalities in Coronary Artery Disease Using Cardiac Magnetic Resonance. Journalof Cardiology and Neuro-cardiovascular Diseases, 2 (6): 2, 2015

7- CROFT P.E., STROUT T.D., KRING R.M., DIRECTOR L., VASAIWALA S.C. and MACKENZIE D.C.: WAMAMI: Emergency physicians can accurately identify wall motion abnormalities in acute myocardialinfarction. The American journal of Emergency Medicine, 37 (12): 2224- 8, 2019.

8- AL-GHARBAWI H.S. and SAEED B.N.: Correlation between regional wall motion abnormalities via 2dimensional echocardiography, and coronary angiographic findings. Iraqi Academic Scientific Journal, 11: 630-4, 2012.

9- SHIVPUJE ANJALI V. and PAGE S.J.: Echocardiographic assessment of left ventricular function in patients of acute myocardial infarction. International Journal of Advances in Medicine, 4 (4): 926, 2017.

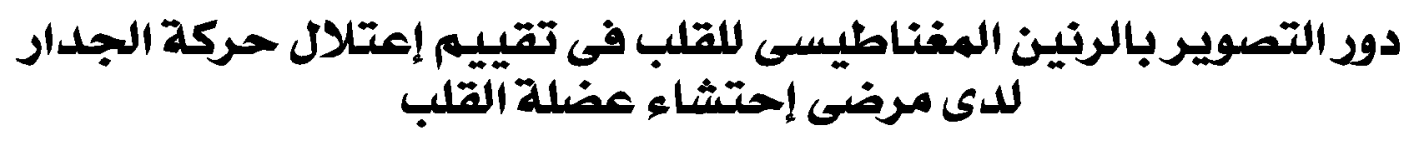

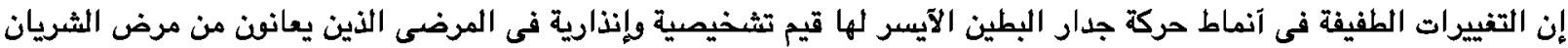

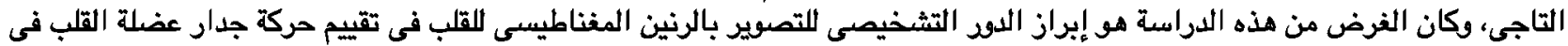
مرضى إحتشاء عضلة القلب. 